\title{
Exploring the impact of regional variation on outcome prioritisation in core outcome set development: a case study in the field of gastric cancer surgery
}

Bilal Alkhaffaf ( $\square$ bilal.alkhaffaf@srft.nhs.uk)

Salford University NHS Foundation Trust

Aleksandra Metryka

Manchester University NHS Foundation Trust

Jane M Blazeby

University of Bristol

Anne-Marie Glenny

University of Manchester

Paula R Williamson

University of Liverpool

lain A Bruce

Manchester University NHS Foundation Trust

\section{Research Article}

Keywords: Core outcome set, Surgical Oncology, Gastric Cancer, Delphi Survey

Posted Date: January 4th, 2021

DOI: https://doi.org/10.21203/rs.3.rs-140027/v1

License: (-) (1) This work is licensed under a Creative Commons Attribution 4.0 International License. Read Full License 


\section{Abstract}

Background

International stakeholder participation is important in the development of core outcome sets (COS). Stakeholders, however, may value health outcomes differently when regional differences are considered. Here, we explore how region, health income and participant characteristics influence prioritisation of outcomes during development of a COS for gastric cancer surgery trials (the GASTROS study).

\section{Methods}

952 participants from 55 countries participating in a Delphi survey during COS development were eligible for inclusion. Recruits were grouped according to region (East or West), country income classification (high and low-to-middle income) and other characteristics (e.g. patients; age, sex, time since surgery, mode of treatment, surgical approach and healthcare professionals; clinical experience). Groups were compared with respect to how they categorised outcomes ('consensus in', 'consensus out', 'no consensus'). Outcomes categorised as 'consensus in' or 'consensus out' by all 3 stakeholder groups would be automatically included in or excluded from the cos respectively.

\section{Results}

In total, 13 outcomes were categorised 'consensus in', 13 'consensus out' and 31 'no consensus'. There was little variation in prioritisation of outcomes by stakeholders from Eastern or Western countries and high or low-to-middle income countries. There was little variation in outcome prioritisation within either health professional or patient groups.

\section{Conclusion}

Our study suggests that there is little variation in opinion within stakeholder groups when participant region and other characteristics are considered. This finding may help COS developers when designing their Delphi surveys and recruitment strategies. Further work across other clinical fields is needed before broad recommendations can be made.

\section{Introduction}

A core outcome set (COS) is an agreed minimum group of critically important outcomes which should be reported by all trials within a research field ${ }^{1}$. The GASTROS study (www.gastrosstudy.org) aims to develop a COS in the field of gastric cancer surgery to promote uniform reporting of important outcomes and facilitate evidence synthesis ${ }^{2}$. This is necessary as there is significant variation and heterogeneity in this field with respect to reporting and measurement of outcomes ${ }^{3}$. Furthermore, the outcomes chosen by researchers to report in surgical trials for gastric cancer often do not reflect the priorities held by patients ${ }^{4}$. For this reason, the GASTROS study has sought consensus between patients and healthcare professionals with respect to outcome selection.

Delphi surveys and consensus meetings are commonly used methodologies in the development of $\operatorname{CoS}^{1,5}$. Delphi surveys ask participants deemed by the study group to hold an important perspective (key stakeholders) to prioritise outcomes and achieve consensus. The completed Delphi survey often informs and influences discussions during a subsequent consensus meeting, with the aim of resolving uncertainties regarding prioritisation and ratifying the final composition of the COS. Clear recruitment strategies for Delphi surveys are an important consideration. If recruitment does not result in representative stakeholder groups, there is a risk that the results of the Delphi may not be valid ${ }^{6}$. This is particularly important in international COS where significant regional and cultural differences may influence the results ahead of a consensus meeting and, ultimately, the final COS.

Ensuring stakeholder groups are representative can be a challenging task. There is a need to consider many factors including the incidence of the disease, treatment protocols, international variation in healthcare systems and values and socio-economic issues. In the case of curative surgery for gastric cancer it is known that practice varies worldwide (e.g. how surgery is carried out and the extent of resection) and typically surgeons value different outcomes to patients ${ }^{4}$. There is therefore a need to explore these issues to understand how key stakeholders are selected for survey participation. In the GASTROS study 952 participants were recruited to a Delphi survey ( 268 were patients, 445 surgeons and 239 nurses) from 55 countries. It was therefore possible to explore how stakeholder charachteristics influenced outcome prioritisation.

This study had two main objectives:

1. To describe the characteristics of Delphi participants and explore their possible influence on the prioritisation of outcomes within stakeholder groups.

2. To explore whether there were regional differences across all stakeholder groups with respect to the categorisation of outcomes.

\section{Methods}

This was an analysis of registration data supplied by Delphi survey participants as part of the GASTROS study. Details of the scope, objectives and methodology of the study have been previously described ${ }^{2-4}$. In summary, participants were asked to score outcomes in terms of importance. The results of the Delphi survey informed discussions in a consensus meeting where final recommendations were made regarding which outcomes to include in the COS.

\subsection{Stakeholder selection and baseline information}


The GASTROS study sought to involve key stakeholders - patients, surgeons, and oncology nurses - to identify a COS for surgical trials in gastric cancer. Our guiding principle has been to promote the 'patient voice' as they are the beneficiaries of trials in this field and have all-important 'lived experience'. The patient voice has previously been shown to be under-represented in COS development ${ }^{7}$. Surgeons provide a clinical perspective and the experience of treating large volumes of patients. Oncology nurses were invited to participate given their central roles as care-givers, patient advocates and core members of the clinical team.

Participation in the Delphi survey was open to all interested stakeholders who fulfilled the following criteria:

- Surgeons who had completed their training and routinely treat gastric cancer.

- Oncology nurses with a recognised proportion of their role involved in the care and follow-up of gastric cancer patients.

- Patients who have undergone surgical resection for gastric cancer with the intention of cure.

There is no sample size requirement for Delphi surveys. To be able to demonstrate the enrolment of a broad and representative range of stakeholders, participants were asked to provide the information listed below:

Patients:

- Age

- Sex

- Surgical approach (laparoscopic or open)

- Type of gastrectomy (total or partial)

- Modality of treatment (surgery alone or a combination of surgery and chemotherapy or radiotherapy)

- Time since surgery

Surgeons:

- Experience (number of gastrectomies undertaken)

Nurses:

- Experience (years of service)

These datapoints were developed based on information that was likely to be readily known to participants and the expert opinion of the GASTROS study management group (SMG) with respect to important factors that may influence outcomes or perspectives. In the context of patients, different health outcomes, such as complications and survival, may impact their lived experience and ultimately how outcomes are prioritised. Similarly, as clinical experience changes with time, there may be a greater exposure to and therefore appreciation of the impact or importance of longer-term consequences of surgery.

Additionally, all participants were asked to provide their country of residence so that regional differences could be considered. Participants were categorised into 'Eastern' or 'Western' countries (figure 1) and 'high-income' or 'low- to medium-income countries' as defined by the Organisation for Economic Cooperation and Development's Development Assistance Committee ${ }^{8}$. Eastern countries were defined as those within East Asia, South East Asia, and Eastern Russia, and included China, Japan, South Korea, Thailand, Vietnam, Malaysia, and Singapore ${ }^{9}$. Western countries were defined as those from Western Europe, North America, Australia, and New Zealand ${ }^{10}$. Contrasting between the 'East' and 'West' is of particular importance to gastric cancer given the differences in incidence, pathology, treatment and outcome. It was hypothesised that these differences in approach and survival may influence how stakeholders in these regions prioritise different health outcomes which could be examined further in this study ${ }^{11,12}$. Similarly, health priorities may be influenced by resource availability as categorised by country income.

\subsection{Scoring of outcomes in the Delphi survey and categorisation of outcomes}

A list of 56 outcomes identified from previous trials and patient interviews ${ }^{3,4}$ were presented to survey participants who were asked to rate each outcome on a scale of importance (1-3: not important, 4-6: important, 7-9: critically important). Patients, surgeons, and nurses group ratings were considered separately to ensure that each group had an equal voice. Participants had the opportunity to suggest further outcomes that they believed had not been presented in round 1. One additional new outcome suggested by participants in round 1 was identified and after consideration by the SMG was presented to participants for scoring in round 2. Therefore, a total of 57 outcomes were presented in round 2 where, for each outcome, participants were shown the scores from each stakeholder group, and given the opportunity to change their rating if they wished.

After two rounds of rating, outcomes were categorised as follows:

- To be included in the cOS ('consensus in')

- To be excluded from the COS ('consensus out')

- 'No consensus' reached i.e. no decision reached as to whether the outcome should be included in of excluded from the COS. 
Criteria for categorising outcomes was set a priori by the SMG and based on established COS methodology ${ }^{1}$. If an outcome was rated 7-9 (critically important) by $70 \%$ or more of a stakeholder group and 1-3 (not important) by no more than $15 \%$ of the group, then the consensus amongst that group was that the outcome should be included in the COS. If an outcome was rated 7-9 (critically important) by less than 50\% of the group, the consensus amongst that group was for the outcome to be excluded from the COS. Unanimous agreement amongst all three stakeholder groups was required for inclusion in, or exclusion from, the COS. Any other combination resulted in the outcome being placed in the 'no consensus' category and was discussed at a pre-planned consensus meeting to finalise the COS.

\subsection{Data analysis and interpretation}

In round 1, participants completing $50 \%$ or more of the Delphi survey were included in the round 1 analysis and invited to participate in round 2 . Likewise, participants completing $50 \%$ or more of the survey in round 2 were included in the round 2 analysis. For the purpose of this present analysis, participants were placed into 'sub-groups' according to the registration data they submitted (e.g. patient treatment type, surgeon experience etc) to examine the differences in outcome scoring. The following analyses were performed after 2 rounds of ratings:

1. The proportion of participants scoring each outcome as 'critically important' (score 7-9). This analysis approach was chosen as these figures were presented in the consensus meeting discussing results from the Delphi survey.

2. The consensus opinion of each sub-group with respect to whether the outcome should be 'included' in the COS, 'excluded' from the COS or whether 'no consensus' could be reached. These categorisations were compared against the overall 'in', 'out' and 'no consensus' categorisations by each stakeholder group (patients, surgeons and nurses) which was presented to the consensus meeting participants.

Participants not providing demographic data during registration were excluded from the sub-group analyses. When exploring differences in prioritisation, particular focus was placed on outcomes that were categorised as 'consensus in' by one sub-group and 'consensus out' by another.

To examine the possible influence of attrition bias between rounds, the characteristics of stakeholders participating in both rounds were compared to those who only completed round 1. A descriptive analysis was undertaken, and the Chi squared test applied to examine for statistically significant differences at the 0.05 level.

\subsection{Ethical Approval}

The study was given ethical approval by the North West - Greater Manchester East Research Ethics Committee (18/NW/0347) and governance approvals by Manchester University Hospitals NHS Foundation Trust.

\section{Results}

\subsection{Overview}

The characteristics of participants included in the analysis and attrition rates are summarised in table 1. After 2 rounds of voting, agreement was reached amongst all three stakeholder groups to include 13 outcomes and exclude 13 outcomes from the Cos, leaving 31 no consensus outcomes for discussion at the consensus meeting.

\subsection{Patients}

A summary of outcomes categorised for 'inclusion' by at least one patient sub-group after 2 rounds of voting is presented in table 2 . Thirty outcomes were categorised for inclusion in the COS by at least one subgroup. Four outcomes were simultaneously categorised both for 'inclusion' and 'exclusion' by different subgroups. None of the outcomes categorised for automatic inclusion by all stakeholder groups were voted 'consensus out' by any patient sub-group. Seven outcomes were categorised for inclusion in the COS by all patient subgroups.

\subsection{Surgeons}

Table 3 summarises and compares outcomes categorised for inclusion by at least one surgeon sub-group after 2 rounds of voting. Twenty-one outcomes were categorised for inclusion by at least one subgroup. No outcomes were simultaneously categorised both for 'inclusion' and 'exclusion' by different subgroups. Twelve outcomes were categorised by all surgeon subgroups for inclusion.

\subsection{Nurses}

Table 4 summarises and compares the outcomes categorised for inclusion by at least one nurse sub-group after 2 rounds of voting. Twenty-two outcomes were categorised for inclusion by at least one subgroup. Five outcomes were simultaneously categorised both for 'inclusion' and 'exclusion' by different subgroups. None of the outcomes categorised for automatic inclusion by all stakeholder groups were voted 'consensus out' by any nurse sub-group. Ten outcomes were categorised by all nurses' subgroups for inclusion. 


\subsection{Regional variations}

Table 5 details the final categorisation list of outcomes which was presented to participants at the consensus meeting. This is compared to alternative outcome categorisation lists based on the region and country income differences described above. Consensus agreement to include 8 and exclude 7 outcomes was reached across all regional sub-groups. No outcomes were simultaneously categorised as 'consensus in' and 'consensus out' across different regional sub-groups.

Table 1 Demographic characteristics of participants included in analysis of round 1 and 2 scores.

\begin{tabular}{|c|c|c|c|c|c|c|}
\hline Stakeholder Group & Variable & Sub-Group & Total & $\begin{array}{l}\text { Completed round } 1 \text { only } \\
(\%)^{*}\end{array}$ & $\begin{array}{c}\text { Completed both rounds } \\
(\%)^{*}\end{array}$ & p value \\
\hline \multirow[t]{18}{*}{ Patients } & All & - & 268 & 84 & 184 & \\
\hline & \multirow[b]{2}{*}{ Age } & $<60$ & & $38(45)$ & $77(42)$ & \multirow[t]{2}{*}{0.69} \\
\hline & & $>=60$ & & $46(55)$ & $107(58)$ & \\
\hline & \multirow[b]{2}{*}{ Sex } & Male & & $52(62)$ & $101(55)$ & \multirow[t]{2}{*}{0.345} \\
\hline & & Female & & $32(38)$ & $83(45)$ & \\
\hline & \multirow[b]{2}{*}{ Region } & West & & $53(62)$ & $113(74)$ & \multirow[t]{2}{*}{0.461} \\
\hline & & East & & $23(38)$ & $39(26)$ & \\
\hline & \multirow[b]{2}{*}{ Country income } & HIC & & $53(63)$ & $113(61)$ & \multirow[t]{2}{*}{0.792} \\
\hline & & LMIC & & $31(37)$ & 71 (39) & \\
\hline & \multirow[b]{3}{*}{ Years since surgery } & $<1$ year & & $15(19)$ & $30(17)$ & \multirow[t]{3}{*}{0.656} \\
\hline & & 1 to 3 years & & $34(44)$ & $68(39)$ & \\
\hline & & $>3$ years & & $29(37)$ & $75(43)$ & \\
\hline & \multirow[b]{2}{*}{ Surgical approach } & Open & & $70(83)$ & $145(78)$ & \multirow[t]{2}{*}{0.850} \\
\hline & & MIS & & $14(17)$ & $31(22)$ & \\
\hline & \multirow[b]{2}{*}{ Type of surgery } & Total & & $40(49)$ & $78(44)$ & \multirow[t]{2}{*}{0.503} \\
\hline & & Partial & & $42(51)$ & $98(56)$ & \\
\hline & \multirow[b]{2}{*}{ Treatment Modality } & Surgery alone & & $28(34)$ & 69 (39) & \multirow[t]{2}{*}{0.495} \\
\hline & & Multimodal therapy & & $54(66)$ & $110(61)$ & \\
\hline \multirow[t]{8}{*}{ Surgeons } & All & - & 445 & 102 & 343 & \multirow{3}{*}{0.000} \\
\hline & \multirow[b]{2}{*}{ Region } & West & & $33(38)$ & $174(61)$ & \\
\hline & & East & & $53(62)$ & $109(39)$ & \\
\hline & \multirow[b]{2}{*}{ Country income } & HIC & & $45(44)$ & $201(59)$ & \multirow[t]{2}{*}{0.010} \\
\hline & & LMIC & & $57(56)$ & $142(41)$ & \\
\hline & \multirow{3}{*}{ Surgeon experience } & $<50$ & & $21(29)$ & $70(23)$ & \multirow[t]{3}{*}{0.45} \\
\hline & & $50-199$ & & $20(27)$ & $103(34)$ & \\
\hline & & $>200$ & & $32(44)$ & $127(43)$ & \\
\hline \multirow[t]{7}{*}{ Nurses } & All & - & 239 & 104 & 135 & \multirow{3}{*}{0.100} \\
\hline & \multirow[b]{2}{*}{ Region } & West & & $22(35)$ & $40(40)$ & \\
\hline & & East & & $57(65)$ & $61(60)$ & \\
\hline & \multirow[b]{2}{*}{ Country income } & HIC & & $24(23)$ & $46(34)$ & \multirow[t]{2}{*}{0.064} \\
\hline & & LMIC & & $80(77)$ & $89(66)$ & \\
\hline & & $0-5$ years & & $59(57)$ & $59(45)$ & 0.056 \\
\hline & Nurse experience & $>5$ years & & $44(43)$ & $73(55)$ & \\
\hline
\end{tabular}

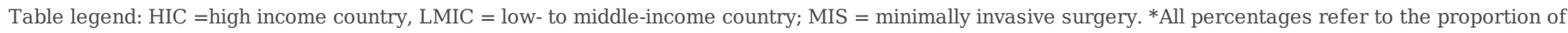
participants from each sub-group completing either round 1 or both rounds.

Table 2 Outcomes categorised for inclusion in the COS by at least one subgroup of patients. 


\begin{tabular}{|c|c|c|c|c|c|c|c|c|c|c|c|c|c|c|c|c|c|c|}
\hline \multirow[b]{2}{*}{ All patients } & \multirow{2}{*}{$\begin{array}{c}\text { Overall } \\
\text { West }\end{array}$} & \multicolumn{2}{|c|}{ Region** } & \multicolumn{2}{|c|}{$\begin{array}{l}\text { Country } \\
\text { Income }\end{array}$} & \multicolumn{2}{|c|}{ Age in years } & \multicolumn{2}{|c|}{ Sex } & \multicolumn{3}{|c|}{ Years since surgery } & \multicolumn{2}{|c|}{$\begin{array}{l}\text { Surgical } \\
\text { approach }\end{array}$} & \multicolumn{2}{|c|}{$\begin{array}{c}\text { Type of } \\
\text { gastrectomy }\end{array}$} & \multicolumn{2}{|c|}{$\begin{array}{c}\text { Treatment } \\
\text { Modality }\end{array}$} \\
\hline & & East & HIC & LMIC & $<60$ & $>=60$ & M & $\mathrm{F}$ & $<1$ & $1-3$ & $>3$ & Open & MIS & Total & Partial & Surgery & $\begin{array}{l}\text { Multi- } \\
\text { modal }\end{array}$ & \\
\hline Outcome & $\mathrm{n}=184$ & $\mathrm{n}=113$ & $n=39$ & $\mathrm{n}=113$ & $\mathrm{n}=71$ & $\mathrm{n}=77$ & $\mathrm{n}=107$ & $\mathrm{n}=101$ & $\mathrm{n}=83$ & $\mathrm{n}=30$ & $\mathrm{n}=68$ & $\mathrm{n}=75$ & $\mathrm{n}=145$ & $\mathrm{n}=31$ & $\mathrm{n}=78$ & $\mathrm{n}=98$ & $\mathrm{n}=69$ & $\mathrm{n}=110$ \\
\hline $\begin{array}{l}\text { 1. Disease-free } \\
\text { survival }^{*}\end{array}$ & 85.4 & 87.0 & 76.3 & 87.0 & 82.9 & 86.7 & 84.5 & 83.0 & 88.5 & 86.2 & 89.4 & 86.1 & 85.9 & 85.7 & 84.9 & 85.6 & 80.3 & 87.9 \\
\hline $\begin{array}{l}\text { 2. Dying from } \\
\text { stomach } \\
\text { cancer* }\end{array}$ & 86.4 & 88.7 & 74.4 & 88.7 & 82.9 & 85.5 & 87.0 & 88.8 & 83.3 & 86.2 & 89.2 & 85.9 & 88.4 & 80.0 & 85.3 & 87.1 & 81.8 & 88.6 \\
\hline $\begin{array}{l}\text { 3. Dying from } \\
\text { any cause }\end{array}$ & 66.7 & 65.0 & 77.8 & 65.0 & 69.1 & 72.6 & 62.2 & 59.6 & 75.3 & 77.8 & 63.6 & 66.2 & 67.2 & 63.0 & 65.3 & 66.3 & 71.4 & 62.1 \\
\hline $\begin{array}{l}\text { 4. Surgery- } \\
\text { related death* }\end{array}$ & 84.0 & 86.9 & 72.2 & 86.9 & 79.4 & 76.7 & 89.2 & 80.2 & 88.6 & 81.5 & 85.1 & 88.7 & 86.2 & 73.3 & 82.7 & 84.9 & 79.7 & 86.8 \\
\hline $\begin{array}{l}7 . \\
\text { Anastomotic } \\
\text { complications* }\end{array}$ & 76.7 & 80.0 & 74.4 & 80.0 & 71.8 & 74.3 & 78.4 & 75.0 & 78.8 & 82.8 & 77.6 & 74.3 & 76.1 & 74.2 & 69.3 & 80.9 & 74.6 & 76.9 \\
\hline $\begin{array}{l}\text { 8. Gastro- } \\
\text { intestinal } \\
\text { functional } \\
\text { problems }\end{array}$ & 72.8 & 85.3 & 71.8 & 85.3 & 53.5 & 69.7 & 75.0 & 66.0 & 81.3 & 75.9 & 63.2 & 80.6 & 70.2 & 77.4 & 70.1 & 72.6 & 67.7 & 74.5 \\
\hline $\begin{array}{l}\text { 9. Bowel } \\
\text { Complications }\end{array}$ & 71.8 & 80.0 & 76.9 & 80.0 & 59.2 & 65.8 & 76.2 & 66.0 & 79.0 & 75.9 & 64.7 & 76.7 & 69.7 & 74.2 & 67.5 & 72.9 & 68.2 & 72.7 \\
\hline $\begin{array}{l}\text { 12. Multiple } \\
\text { organ failure* }\end{array}$ & 86.4 & 87.9 & 86.5 & 87.9 & 84.1 & 87.8 & 85.3 & 86.7 & 85.9 & 86.2 & 86.4 & 85.7 & 87.8 & 79.3 & 83.8 & 87.2 & 80.3 & 89.5 \\
\hline $\begin{array}{l}\text { 16. Hepatic } \\
\text { Complications }\end{array}$ & 62.4 & 65.0 & 73.7 & 65.0 & 57.1 & 71.6 & 54.5 & 52.1 & 73.4 & 78.6 & 52.3 & 60.9 & 61.0 & 62.1 & 60.8 & 59.3 & 67.2 & 56.4 \\
\hline $\begin{array}{l}\text { 17. Pancreatic } \\
\text { Complications }\end{array}$ & 70.3 & 75.5 & 73.7 & 75.5 & 61.4 & 74.0 & 66.7 & 63.8 & 76.9 & 82.1 & 57.8 & 72.5 & 68.9 & 69.0 & 67.1 & 69.2 & 68.7 & 69.0 \\
\hline \multirow{2}{*}{$\begin{array}{l}\text { 18. Abdominal } \\
\text { Collection }\end{array}$} & 71.5 & 72.3 & 82.1 & 72.3 & 70.4 & 71.6 & 71.4 & 65.3 & 79.2 & 75.9 & 70.8 & 67.2 & 69.2 & 77.4 & 63.9 & 75.0 & 73.1 & 69.0 \\
\hline & $\begin{array}{c}\text { All } \\
\text { patients }\end{array}$ & West & East & HIC & LMIC & $<60$ & $>=60$ & $\mathrm{M}$ & $\mathrm{F}$ & $<1$ & $1-3$ & $>3$ & Open & MIS & Total & Partial & Surgery & $\begin{array}{l}\text { Multi- } \\
\text { modal }\end{array}$ \\
\hline $\begin{array}{l}20 . \\
\text { Nutritional } \\
\text { Effects* }\end{array}$ & 73.8 & 77.7 & 69.2 & 77.7 & 66.2 & 75.3 & 71.7 & 69.0 & 78.3 & 73.3 & 72.1 & 75.7 & 72.9 & 71.0 & 75.6 & 70.1 & 69.1 & 74.5 \\
\hline $\begin{array}{l}21 . \\
\text { Recurrence of } \\
\text { Cancer* }^{*}\end{array}$ & 92.2 & 95.4 & 84.6 & 95.4 & 85.9 & 88.0 & 94.3 & 92.0 & 91.3 & 93.1 & 88.1 & 95.9 & 91.5 & 90.3 & 88.0 & 93.8 & 88.1 & 93.5 \\
\hline $\begin{array}{l}\text { 22. Renal } \\
\text { complications }\end{array}$ & 70.0 & 80.0 & 65.8 & 80.0 & 54.3 & 66.2 & 71.7 & 67.0 & 72.4 & 82.1 & 53.8 & 80.3 & 69.2 & 65.5 & 64.8 & 71.4 & 67.2 & 71.4 \\
\hline $\begin{array}{l}\text { 23. Urinary } \\
\text { complications }\end{array}$ & 58.1 & 65.7 & 57.9 & 65.7 & 45.7 & 54.2 & 60.0 & 54.2 & 61.8 & 64.3 & 40.0 & 70.6 & 57.8 & 51.7 & 50.0 & 60.9 & 56.1 & 59.4 \\
\hline $\begin{array}{l}25 . \\
\text { Respiratory } \\
\text { complications }\end{array}$ & 69.5 & 67.0 & 66.7 & 67.0 & 73.2 & 70.3 & 68.9 & 70.7 & 67.9 & 75.0 & 73.1 & 63.4 & 71.2 & 56.7 & 60.8 & 73.7 & 68.2 & 68.9 \\
\hline $\begin{array}{l}\text { 27. Cerebro- } \\
\text { vascular } \\
\text { complications }\end{array}$ & 77.6 & 81.0 & 68.4 & 81.0 & 72.9 & 68.6 & 84.0 & 80.9 & 73.7 & 75.0 & 72.3 & 84.8 & 78.0 & 73.3 & 69.0 & 82.4 & 71.2 & 82.8 \\
\hline $\begin{array}{l}\text { 28. Thrombo- } \\
\text { embolic } \\
\text { complications }\end{array}$ & 76.7 & 80.4 & 63.2 & 80.4 & 71.4 & 73.2 & 79.2 & 78.9 & 74.0 & 71.4 & 73.8 & 82.4 & 79.9 & 60.0 & 73.6 & 77.2 & 66.7 & 84.2 \\
\hline 29. Bleeding* & 72.3 & 67.6 & 76.9 & 67.6 & 78.9 & 77.5 & 68.6 & 67.0 & 78.9 & 75.0 & 72.7 & 69.1 & 73.3 & 66.7 & 70.0 & 71.6 & 72.7 & 70.6 \\
\hline
\end{tabular}




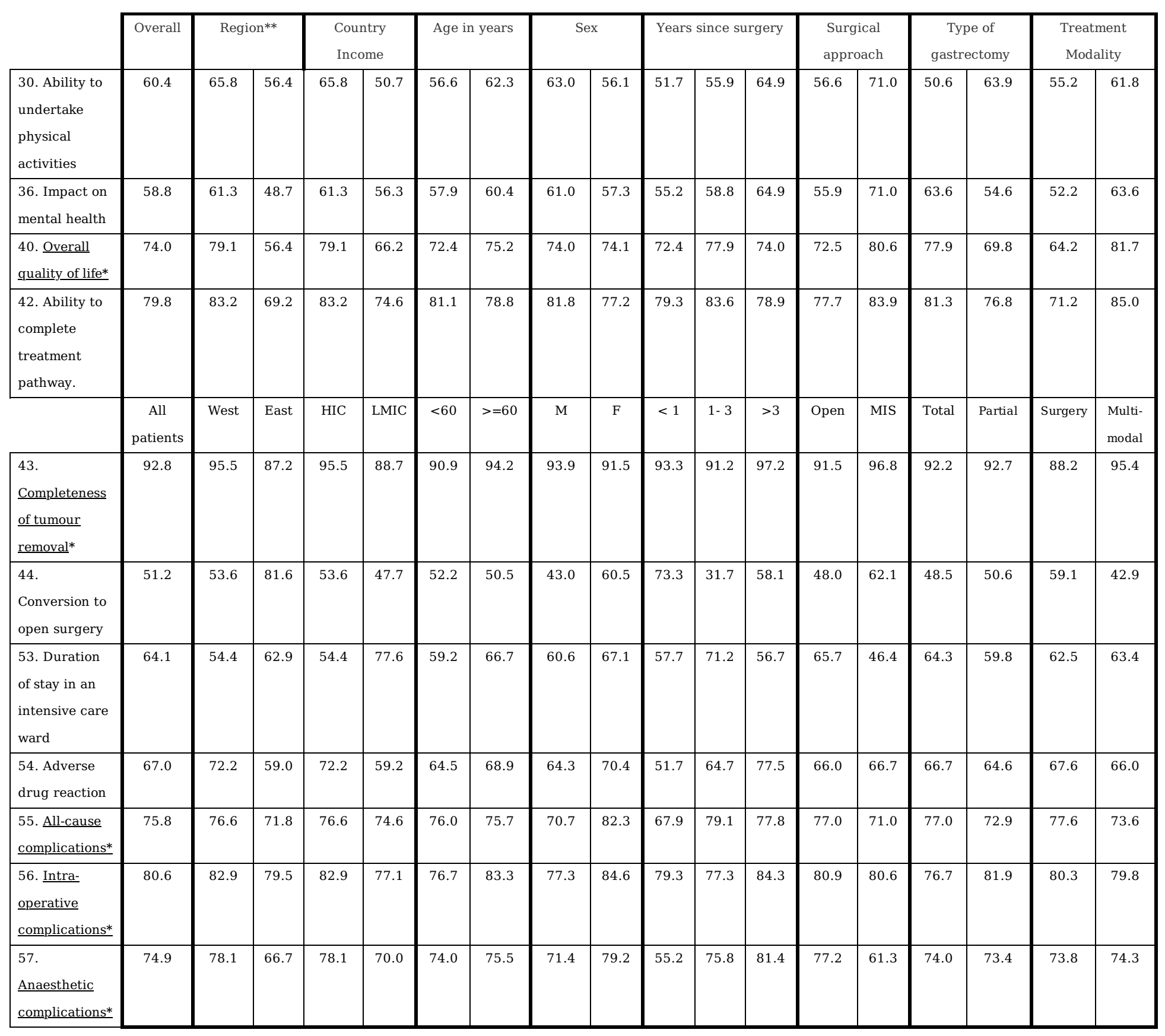

Values are the percentage of participants voting the outcome as critically important (score 7-9).

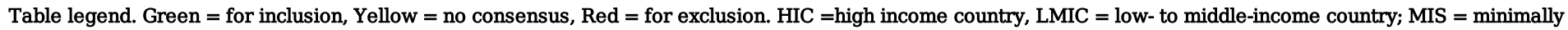

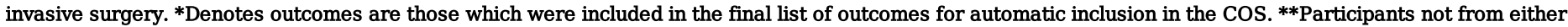
Western or Eastern countries were excluded from this analysis.

Table 3 Outcomes categorised for inclusion in the COS by at least one subgroup of surgeons. 


\begin{tabular}{|l|c|c|c|c|c|c|c|c|}
\cline { 2 - 9 } \multicolumn{1}{c|}{} & Overall & \multicolumn{2}{|c|}{ Region** } & \multicolumn{2}{c|}{ Country Income } & \multicolumn{2}{c|}{ Cases performed } \\
\cline { 2 - 9 } \multicolumn{1}{c|}{} & All surgeons & West & East & HIC & LMIC & $<50$ & $\mathbf{5 0 - 1 9 9}$ & $>\mathbf{2 0 0}$ \\
\hline Outcome & $\mathrm{n}=343$ & $\mathrm{n}=174$ & $\mathrm{n}=109$ & $\mathrm{n}=201$ & $\mathrm{n}=142$ & $\mathrm{n}=70$ & $\mathrm{n}=103$ & $\mathrm{n}=127$ \\
\hline 1. Disease-free survival* & 97.7 & 97.7 & 98.1 & 98.0 & 97.2 & 95.7 & 99.0 & 97.6 \\
\hline 2. Dying from stomach cancer* & 96.5 & 97.7 & 95.4 & 96.0 & 97.2 & 95.7 & 95.1 & 96.9 \\
\hline 4. Surgery-related death* & 96.8 & 96.6 & 99.1 & 97.5 & 95.8 & 94.3 & 96.1 & 98.4 \\
\hline 7. Anastomotic complications* & 95.3 & 95.4 & 95.4 & 96.0 & 95.1 & 95.7 & 94.2 & 96.1 \\
\hline 8. Gastro-intestinal functional problems & 74.9 & 75.3 & 70.6 & 75.1 & 76.1 & 82.9 & 76.7 & 67.7 \\
\hline 12. Multiple organ failure* & 81.3 & 81.0 & 78.9 & 81.1 & 80.9 & 75.7 & 83.5 & 81.7 \\
\hline 18. Abdominal Collection & 73.4 & 75.1 & 67.0 & 74.5 & 73.2 & 71.4 & 69.9 & 78.7 \\
\hline 20. Nutritional Effects* & 72.8 & 74.6 & 66.1 & 73.5 & 73.9 & 77.1 & 75.7 & 69.3 \\
\hline 21. Recurrence of Cancer* & 97.7 & 99.4 & 95.4 & 99.0 & 96.5 & 97.1 & 100.0 & 97.6 \\
\hline 25. Respiratory complications & 66.5 & 70.1 & 59.6 & 70.6 & 62.0 & 65.7 & 67.0 & 70.1 \\
\hline 28. Thrombo-embolic complications & 64.1 & 63.2 & 60.6 & 63.2 & 65.5 & 61.4 & 59.2 & 70.9 \\
\hline 29. Bleeding* & 87.5 & 84.5 & 95.4 & 86.1 & 90.1 & 81.4 & 85.4 & 92.9 \\
\hline 30. Ability to undertake physical activities & 66.4 & 71.8 & 59.6 & 69.7 & 63.4 & 65.7 & 70.9 & 66.9 \\
\hline 40. Overall quality of life* & 86.5 & 93.1 & 75.9 & 90.0 & 82.3 & 91.4 & 87.4 & 85.7 \\
\hline 42. Ability to complete treatment pathway. & 78.6 & 86.2 & 61.1 & 82.6 & 73.6 & 87.0 & 74.8 & 75.4 \\
\hline 43. Completeness of tumour removal* & 97.4 & 98.3 & 97.2 & 98.5 & 95.7 & 91.4 & 99.0 & 99.2 \\
\hline 49. Readmission to hospital & 78.9 & 78.7 & 82.4 & 78.6 & 80.9 & 80.0 & 81.6 & 81.0 \\
\hline 51. Need for an additional intervention. & 75.4 & 82.8 & 59.3 & 81.6 & 66.7 & 78.6 & 78.6 & 71.4 \\
\hline 55. All-cause complications* & 81.2 & 81.5 & 84.3 & 83.0 & 79.4 & 81.4 & 76.7 & 88.1 \\
\hline 56. Intra-operative complications* & 91.5 & 88.4 & 93.5 & 89.5 & 93.6 & 91.4 & 92.2 & 92.9 \\
\hline 57. Anaesthetic complications* & 70.5 & 70.3 & 71.0 & 70.4 & 70.7 & 68.6 & 66.0 & 75.2 \\
\hline
\end{tabular}

Values are the percentage of participants voting the outcome as critically important (score 7-9).

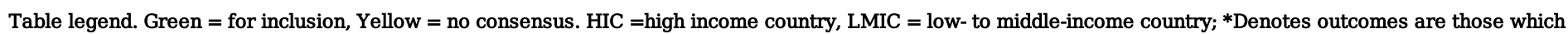

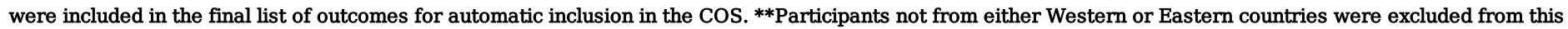
analysis.

Table 4 Outcomes categorised for inclusion in the COS by at least one subgroup of nurses. 


\begin{tabular}{|c|c|c|c|c|c|c|c|}
\hline & \multirow{2}{*}{\begin{tabular}{|c|} 
Overall \\
All nurses
\end{tabular}} & \multicolumn{2}{|c|}{ Region** } & \multicolumn{2}{|c|}{ Country Income } & \multicolumn{2}{|c|}{ Experience in years } \\
\hline & & West & East & HIC & LMIC & $0-5$ years & $>5$ \\
\hline Outcome & $\mathrm{n}=135$ & $\mathrm{n}=40$ & $\mathrm{n}=61$ & $\mathrm{n}=46$ & $\mathrm{n}=89$ & $\mathrm{n}=59$ & $\mathrm{n}=73$ \\
\hline 1. Disease-free survival* & 85.1 & 92.5 & 85.2 & 93.5 & 80.9 & 81.4 & 89.0 \\
\hline 2. Dying from stomach cancer* & 80.0 & 90.0 & 72.1 & 91.3 & 74.2 & 74.6 & 83.6 \\
\hline 3. Dying from any cause & 63.4 & 64.1 & 70.5 & 64.4 & 65.2 & 58.6 & 71.2 \\
\hline 4. Surgery-related death & 77.6 & 95.0 & 65.6 & 93.5 & 69.3 & 72.9 & 81.9 \\
\hline 7. Anastomotic complications* & 84.4 & 97.5 & 82.0 & 97.8 & 76.4 & 79.7 & 89.0 \\
\hline 8. Gastro-intestinal functional problems & 69.6 & 90.0 & 65.6 & 89.1 & 57.3 & 59.3 & 75.3 \\
\hline 12. Multiple organ failure* & 79.9 & 82.5 & 78.3 & 84.8 & 78.4 & 83.1 & 79.2 \\
\hline 13. Pain & 59.3 & 85.0 & 59.0 & 87.0 & 44.9 & 49.2 & 65.8 \\
\hline 18. Abdominal Collection & 65.9 & 65.0 & 67.2 & 69.6 & 61.8 & 49.2 & 76.7 \\
\hline 19. Other infections & 61.2 & 55.0 & 70.0 & 58.7 & 61.4 & 54.2 & 65.3 \\
\hline 20. Nutritional Effects* & 74.8 & 87.5 & 77.0 & 87.0 & 66.3 & 69.5 & 76.7 \\
\hline 21. Recurrence of Cancer* & 88.0 & 97.5 & 86.9 & 97.8 & 82.8 & 84.5 & 90.3 \\
\hline 26. Wound complications & 67.4 & 62.5 & 73.8 & 63.0 & 67.4 & 67.8 & 64.4 \\
\hline 29. Bleeding* & 80.7 & 72.5 & 85.2 & 76.1 & 82.0 & 79.7 & 80.8 \\
\hline 30. Ability to undertake physical activities & 56.3 & 72.5 & 54.1 & 73.9 & 46.1 & 54.2 & 56.2 \\
\hline 36. Impact on mental health & 54.5 & 70.0 & 48.3 & 71.7 & 44.3 & 54.2 & 52.8 \\
\hline 40. Overall quality of life* & 70.4 & 90.0 & 67.2 & 89.1 & 59.6 & 61.0 & 76.7 \\
\hline 42. Ability to complete treatment pathway. & 65.9 & 77.5 & 60.7 & 78.3 & 58.4 & 54.2 & 75.3 \\
\hline 43. Completeness of tumour removal* & 87.3 & 100.0 & 86.9 & 97.8 & 82.0 & 83.1 & 91.8 \\
\hline 49. Readmission to hospital & 69.9 & 77.5 & 68.3 & 78.3 & 62.1 & 60.3 & 73.6 \\
\hline 51. Need for an additional intervention. & 56.7 & 75.0 & 48.3 & 76.1 & 45.5 & 44.1 & 63.9 \\
\hline 52. Need for pain relief & 68.4 & 72.5 & 72.9 & 73.9 & 63.2 & 57.6 & 74.6 \\
\hline 55. All-cause complications* & 77.9 & 77.5 & 77.2 & 80.4 & 75.3 & 70.2 & 83.1 \\
\hline 56. Intra-operative complications* & 85.4 & 90.0 & 91.1 & 91.3 & 83.3 & 85.7 & 87.3 \\
\hline 57. Anaesthetic complications* & 78.0 & 80.0 & 77.8 & 80.4 & 76.5 & 70.9 & 84.1 \\
\hline
\end{tabular}

Values are the percentage of participants voting the outcome as critically important (score 7-9). Table legend. Green $=$ for inclusion, Red $=$ for exclusion, Yellow $=$ no consensus. HIC =high income country, LMIC = low- to middle-income country; *Denotes outcomes are those which were included in the final list of outcomes for automatic inclusion in the COS. **Participants not from either Western or Eastern countries were excluded from this analysis.

Table 5 Regional differences in consensus on outcomes voted for inclusion or exclusion from the COS by at least 1 subgroup. 


\begin{tabular}{|c|c|c|c|c|c|}
\hline \multirow[b]{2}{*}{ Consensus outcome } & \multirow[t]{2}{*}{ Final List } & \multicolumn{2}{|c|}{ Region** } & \multicolumn{2}{|c|}{ Country Consensus income } \\
\hline & & $\begin{array}{c}\text { West } \\
(\mathrm{n}=327)\end{array}$ & $\begin{array}{c}\text { East } \\
(\mathrm{n}=209)\end{array}$ & $\begin{array}{c}\text { HIC } \\
(n=360)\end{array}$ & $\begin{array}{c}\text { LMIC } \\
(\mathrm{n}=302)\end{array}$ \\
\hline 1. Disease-free survival* & Consensus in & Consensus in & Consensus in & Consensus in & Consensus in \\
\hline 2. Dying from stomach cancer* & Consensus in & Consensus in & Consensus in & Consensus in & Consensus in \\
\hline 4. Surgery-related death & Consensus in & Consensus in & No consensus & Consensus in & No consensus \\
\hline 6. Endocrine complications & Consensus out & Consensus out & No consensus & Consensus out & Consensus out \\
\hline 7. Anastomotic complications* & Consensus in & Consensus in & Consensus in & Consensus in & Consensus in \\
\hline 8. Gastro-Intestinal functional problems & No consensus & Consensus in & No consensus & Consensus in & No consensus \\
\hline 11. Fatigue & Consensus out & Consensus out & Consensus out & Consensus out & Consensus out \\
\hline 12. Multiple organ failure* & Consensus in & Consensus in & Consensus in & Consensus in & Consensus in \\
\hline 14. Surgical Stress Response & Consensus out & Consensus out & No consensus & Consensus out & No consensus \\
\hline 15. Gallbladder complications & No consensus & No consensus & No consensus & No consensus & Consensus out \\
\hline 20. Nutritional Effects & Consensus in & Consensus in & No consensus & Consensus in & No consensus \\
\hline 21. Recurrence of Cancer* & Consensus in & Consensus in & Consensus in & Consensus in & Consensus in \\
\hline 23. Urinary complications & No consensus & No consensus & No consensus & No consensus & Consensus out \\
\hline 24. Post-operative psychosis & Consensus out & Consensus out & Consensus out & Consensus out & Consensus out \\
\hline 29. Bleeding & Consensus in & No consensus & Consensus in & No consensus & Consensus in \\
\hline 31. Insomnia & Consensus out & Consensus out & No consensus & Consensus out & Consensus out \\
\hline 32. Impact on sexual function & Consensus out & Consensus out & Consensus out & Consensus out & Consensus out \\
\hline 33. Ability to eat socially & Consensus out & No consensus & Consensus out & No consensus & Consensus out \\
\hline 34. Ability to Interact socially & Consensus out & No consensus & Consensus out & No consensus & Consensus out \\
\hline 35. Impact of surgery on social and work roles & No consensus & No consensus & Consensus out & No consensus & Consensus out \\
\hline 36. Impact on mental health & No consensus & No consensus & Consensus out & No consensus & No consensus \\
\hline 37. Impact on Physical Appearance & Consensus out & Consensus out & Consensus out & Consensus out & Consensus out \\
\hline 39. Impact on spirituality or faith & Consensus out & Consensus out & Consensus out & Consensus out & Consensus out \\
\hline 40. Overall quality of life & Consensus in & Consensus in & No consensus & Consensus in & No consensus \\
\hline 41. Impact on perception of physical health & No consensus & No consensus & No consensus & No consensus & Consensus out \\
\hline 42. Ability to complete treatment pathway. & No consensus & Consensus in & No consensus & Consensus in & No consensus \\
\hline 43. Completeness of tumour removal* & Consensus in & Consensus in & Consensus in & Consensus in & Consensus in \\
\hline 45. Duration of surgery & No consensus & Consensus out & No consensus & Consensus out & No consensus \\
\hline 46. Wound size & Consensus out & Consensus out & Consensus out & Consensus out & Consensus out \\
\hline 47. Cost & Consensus out & Consensus out & No consensus & Consensus out & No consensus \\
\hline 50. Destination on Discharge & Consensus out & Consensus out & Consensus out & Consensus out & Consensus out \\
\hline 55. All-cause complications* & Consensus in & Consensus in & Consensus in & Consensus in & Consensus in \\
\hline 56. Intra-operative complications* & Consensus in & Consensus in & Consensus in & Consensus in & Consensus in \\
\hline 57. Anaesthetic complications & Consensus in & Consensus in & No consensus & Consensus in & Consensus in \\
\hline
\end{tabular}

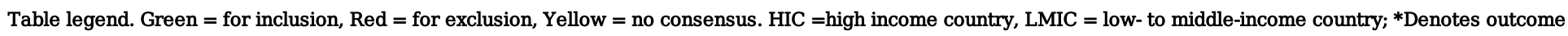
was categorised as for 'inclusion' in COS by all subgroups. **Participants not from either Western or Eastern countries were excluded from this analysis.

\section{Discussion}

The GASTROS study (www.gastrosstudy.org) is the first to bring together healthcare professionals and patients with the purpose of identifying outcomes to include in a COS for surgical trials in gastric cancer. The multi-language survey recruited a broad spectrum of stakeholders with different personal and professional experiences from over 50 countries across 6 continents. We aimed to examine whether certain stakeholder characteristics influenced how outcomes were prioritised and whether there were regional influences also. Our analysis from nearly 1000 survey participants suggested that little variation within the stakeholder groups exists. Similarly, when all stakeholders were categorised according to region or country income, significant differences were not identified. These are important findings which should serve to reassure researchers and patients that the resulting COS has sought and considered international opinion. Furthermore, these findings suggest that priorities within stakeholder groups and across regions are more aligned than may have been previously thought.

\subsection{Planning recruitment to Delphi surveys}


Few studies have previously examined factors which influence how stakeholders prioritise outcomes in the field of COS development. The BRAVO study explored this in the field of breast cancer reconstruction and found that priorities varied within patient and healthcare professional groups ${ }^{6}$. This led them to recommend careful participant selection for Delphi surveys by COS developers. These same differences, however, were not identified in our study. The BRAVO study's healthcare professional stakeholder group was more heterogenous than the groups in this study (breast surgeons, plastic surgeons, nurses and psychologists grouped together) and so these differences may be expected. Furthermore, reconstructive breast surgery is a complex area which covers many different types of procedures. This may also account for the significant variation in outcome prioritisation by patients which was not mirrored in the GASTROS study. Similarly, a COS study in the field of bariatric surgery identified significant variation in outcome prioritisation amongst healthcare professionals ${ }^{13}$. Again, healthcare professionals in this study were heterogenous, which supports our strategy to separate surgeons and nurses into different stakeholder groups.

Achieving the 'correct balance' of representative stakeholders is an important consideration during the design phase. For example, knowledge of the patient demographic and which types of interventions are prevalent within that group, will enable researchers to recruit an appropriate number of stakeholders with those characteristics. With respect to the GASTROS study, the importance of seeking international agreement on core outcomes was identified at the conception stage and subsequently influenced the design of the prioritisation exercise. Our strategy for addressing the significant challenges associated with international involvement included 1) an international working group with regional collaborators, 2) translating surveys and 3) seeking the support of relevant patient and professional groups. Transparent reporting of methodological approaches adopted during cos development are of paramount importance. Ultimately, a coS will only achieve its stated goals if researchers use it. And whilst there are likely several factors which influence the uptake of cos, ensuring researchers have the confidence that the COS is relevant to them and has been developed through a methodologically robust process are likely to be important factors which influence uptake and dissemination ${ }^{14}$.

There are challenges in deciding how to sample participants for a Delphi study. Epidemiological studies, registries and audits provide descriptive regional or national information ${ }^{15-17}$. However, in the case of gastric cancer, these resources are not always complete or available. Consequently, the study team widened the promotion and enrolment into the Delphi to capture as many patients as possible. In our study, we demonstrated that there was not significant variation in outcome prioritisation within stakeholder sub-groups with respect to the characteristics that we examined. Consequently, whilst over 1000 participants were enrolled, it may not have been necessary to recruit such large numbers. This will likely guide our recruitment strategy during future planned stages of work when reviewing the COS and identifying outcome measurement instruments. Our experience may also help guide other COS developers as they consider the number of participants to recruit to their Delphi surveys. However, given some of our findings differed from those in the field of breast surgery reconstruction and bariatric surgery, more work is needed before broad recommendations can be made.

\subsection{Variations within stakeholder groups}

When regional variations across the three stakeholder groups were compared, the greatest differences in prioritisation were observed amongst nurses. For example, in four outcomes (pain, ability to undertake physical exercise, impact on mental health, need for additional intervention) different subgroups of nurses categorised them as 'consensus in' and 'consensus out'. These outcomes seemed less important in LMIC and HIC settings within the nurse group. Understanding the reason for this is likely to be complex. It may be argued that this is simply because nurses are reflecting the importance that patients from these cultures or regions place on these outcomes as similar trends were seen amongst patients. Limited resource in LMIC settings which may affect followup may also play a role in understanding how important longer-term problems are in these regions. Further exploration using qualitative research methods may help understand these differences further.

In examining the differences between patient sub-groups, one would expect to see some differences given the number of characteristics that were examined. Despite this, only two outcomes (urinary complications and conversion to open surgery) were simultaneously categorised as 'consensus in' and 'consensus out' by different sub-groups. This finding suggests that despite the many possible influences on patient experience following gastric cancer surgery, there is not a significant variation in how health related outcomes are prioritised in this group. Surgeons had the greatest concordance with respect to outcome prioritisation. Overall, the observed differences in outcome prioritisation were small within each stakeholder group reassuring researchers using the COS that it is based on the views of a representative cohort of patients and healthcare professionals.

\subsection{Impact of regional variations on outcomes automatically included in COS}

The aim of a COS is to identify outcomes which are critically important across all stakeholder groups participating in the process. In the case of the GASTROS study, an outcome would only be automatically included in the COS if patients, surgeons, and nurses each categorise it 'consensus in'. Ultimately, it is not possible to confidently assess how regional differences may have affected the final categorisation of outcomes which informed the consensus meeting. Participants in round 2 were shown the scores of all stakeholder groups from round 1 before being asked to change their score if they wish. To assess regional differences, Western participants, for example, in round 2 would have needed to see only Western stakeholder group scores from round 1. Furthermore, there are a number of other confounding factors which influence why participants change scores between rounds (see below) further making an analysis of regional impacts challenging.

Despite this, some assessments could be made. No outcomes categorised for automatic inclusion by all three stakeholder groups were categorised for automatic exclusion by a regional sub-group. And no outcomes categorised for automatic exclusion from the COS by all three stakeholder groups were categorised for automatic inclusion by a regional sub-group. This suggests that the regional differences in approach to management or patient outcome may not significantly influence how stakeholders prioritise outcomes

Page $11 / 15$ 
There were two outcomes (gastrointestinal functional problems and ability to complete treatment pathway) which were categorised for automatic inclusion by stakeholders from the West and HIC that were not included in the final list presented to the consensus meeting. Furthermore, some outcomes (surgeryrelated death, nutritional outcomes, bleeding, overall quality of life, anaesthetic complications) did not reach consensus for automatic inclusion by regional sub-groups yet were automatically included when the overall views of stakeholders were considered. This may bring some to the conclusion that different COS should be developed for different regions as some researchers may be collecting outcomes that were not deemed critically important in their region. However, researchers should be cognisant of the fact that their trials are internationally relevant and vitally important to the larger picture where evidence synthesis is concerned. From a different perspective, some researchers may feel aggrieved if outcomes which are critically important in their region are not eventually included in the COS. It is important to emphasise that COS are minimum reporting guidelines and that researchers are encouraged to report additional outcomes that they believe are important.

\subsection{Strengths and Limitations}

Strengths of this study include that it is novel and that is was able to recruit a large number of participants from many countries. However, there are some limitations which should be acknowledged. This was an analysis which was not powered to make definitive conclusions about relationships between subgroups and how outcomes were rated. Therefore, the results should be viewed in this context. Furthermore, the sub-groups examined in this paper were chosen by members of the study team based on their extensive experience in the field of gastric cancer and their understanding of factors which may impact on stakeholder experience, perceptions and subsequently how outcomes may be prioritised. It is possible that other unexplored characteristics impact on how stakeholders prioritise outcomes. In addition, this study did not explore how different characteristics interact with one another to impact on outcome prioritisation (e.g. years since surgery and type of gastrectomy). Doing so would create results which would remove the focus from regional differences and would be difficult to interpret. Furthermore, there were significantly fewer patients from Eastern countries enrolled compared to their Western counterparts. This may have influenced how outcomes were categorised ahead of the consensus meeting. However, due to the interplay of other factors described above, reaching a definite conclusion about the degree of this possible limitation is difficult. This is an area that may benefit from further exploration using qualitative research methods.

Delphi surveys are an established method of reaching consensus in the design of $\cos ^{1}$. They give participants the opportunity to reflect on their ratings from previous rounds before giving a final score. Only after this opportunity should all scores be analysed, and outcomes categorised ahead of the consensus meeting. During the process of rating outcomes in round 2 of the survey, participants are shown the results from each separate stakeholder group in round 1. The topic of why participants change their scores between rounds is an interesting one which has been examined elsewhere ${ }^{18}$. Through our previous analysis we identified that the reasons for changing scores provided by stakeholders were varied, including having the time to reflect on the question being asked, changing their minds on the importance, impact or usefulness of the outcome in question, and changes in personal experience of the outcome. In fact, the influence of other stakeholder ratings as a reason for significantly changing a score in round 2 was cited by only a minority of healthcare professionals and patients.

Another factor which may influence scores between rounds is attrition. Our strategy to keep this as low as possible, alongside other approaches to facilitate international participation in Delphi surveys for COS is a topic which will be described separately. Whilst overall attrition was $30 \%$, the group this affected the most were nurses with nearly $45 \%$ attrition. However, the characteristics of those completing both rounds were not significantly different to those only completing round 1. Likewise, a statistically significant difference was identified in the characteristics of surgeons completing both rounds who were predominantly Western and from HIC compared to the balance of surgeons completing round 1 . It could be argued therefore that retaining a greater number of Eastern and LMIC surgeons may have led to slightly different survey results. However, whilst statistically significant, this difference is unlikely to be clinically significant given that the number of surgeons not participating in round 2 was relatively small.

\subsection{Conclusion}

The GASTROS Delphi survey recruited a broad spectrum of international stakeholders to produce a list of outcomes which should be included or excluded from a COS and others which required further discussion at a consensus meeting. Whilst some regional differences were highlighted, there was little variation within stakeholder groups and between regions with respect to how outcomes were prioritised. This may reassure COS users that the adopted methodology was robust and that the views captured during its development were representative. COS developers should carefully consider the characteristics of Delphi survey participants when planning their recruitment strategy.

\section{References}

1. Williamson PR, Altman DG, Bagley H, et al. The COMET Handbook: version 1.0. Trials. 2017;18(S3):280. doi:10.1186/s13063-017-1978-4

2. Alkhaffaf B, Glenny A-M, Blazeby JM, Williamson P, Bruce IA. Standardising the Reporting of Outcomes in Gastric Cancer Surgery Trials: Protocol for the Development of a Core Outcome Set and Accompanying Outcome Measurement Instrument Set (The GASTROS Study). Trials. 2017;18(1):370. doi:10.1186/s13063-017-2100-7

3. Alkhaffaf B, Blazeby JM, Williamson PR, Bruce IA, Glenny A-M. Reporting of outcomes in gastric cancer surgery trials: a systematic review. BMJ Open. 2018;8(10):e021796. doi:10.1136/BMJOPEN-2018-021796

4. Alkhaffaf B, Blazeby JM, Bruce IA, Morris RL. Patient priorities in relation to surgery for gastric cancer: qualitative interviews with gastric cancer surgery patients to inform the development of a core outcome set. BMJ Open. 2020;10(2):e034782. doi:10.1136/bmjopen-2019-034782

Page $12 / 15$ 
5. Williamson PR, Altman DG, Blazeby JM, et al. Developing core outcome sets for clinical trials: issues to consider. Trials. 2012;13(1):132. doi:10.1186/1745-6215-13-132

6. Potter S, Brookes ST, Holcombe C, Ward JA, Blazeby JM. Exploring methods the for selection and integration of stakeholder views in the development of core outcome sets: A case study in reconstructive breast surgery. Trials. 2016;17(1):1-11. doi:10.1186/s13063-016-1591-y

7. Gargon E, Gurung B, Medley N, et al. Choosing Important Health Outcomes for Comparative Effectiveness Research: A Systematic Review. Scherer RW, ed. PLoS One. 2014;9(6):e99111. doi:10.1371/journal.pone.0099111

8. World Bank Country and Lending Groups - World Bank Data Help Desk. https://datahelpdesk.worldbank.org/knowledgebase/articles/906519-worldbank-country-and-lending-groups. Accessed May 5, 2020.

9. Far East - Wikipedia. https://en.wikipedia.org/wiki/Far_East. Accessed June 28, 2020.

10. Western world - Wikipedia. https://en.wikipedia.org/wiki/Western_world. Accessed June 28, 2020.

11. Yamamoto M, Rashid OM, Wong J. Surgical management of gastric cancer: The East vs. West perspective. J Gastrointest Oncol. 2015;6(1):79-88. doi:10.3978/j.issn.2078-6891.2014.097

12. Chan WL, Lam KO, Lee VHF, et al. Gastric Cancer - From Aetiology to Management: Differences Between the East and the West. Clin Oncol. 2019;31(8):570-577. doi:10.1016/j.clon.2019.05.012

13. Coulman KD, Howes N, Hopkins J, et al. A Comparison of Health Professionals' and Patients' Views of the Importance of Outcomes of Bariatric Surgery. Obes Surg. 2016;26(11):2738-2746. doi:10.1007/s11695-016-2186-0

14. Kirkham JJ, Bracken M, Hind L, Pennington K, Clarke M, Williamson PR. Industry funding was associated with increased use of core outcome sets. J Clin Epidemiol. 2019;115:90-97. doi:10.1016/j.jclinepi.2019.07.007

15. Bang Y-J, Yalcin S, Roth A, et al. Registry of gastric cancer treatment evaluation (REGATE): I baseline disease characteristics. Asia Pac J Clin Oncol. 2014;10(1):38-52. doi:10.1111/ajco.12112

16. Messager M, De Steur WO, Van Sandick JW, et al. Variations among 5 European countries for curative treatment of resectable oesophageal and gastric cancer: A survey from the EURECCA Upper GI Group (EUropean REgistration of Cancer CAre). Eur J Surg Oncol. 2016;42(1):116-122. doi:10.1016/j.ejso.2015.09.017

17. Michalowski J, Salvador A, Napper R. Commissioned by Healthcare Quality Improvement Partnership National Oesophago-Gastric Cancer Audit 2018 An Audit of the Care Received by People with Oesophago-Gastric Cancer in England and Wales 2018 Annual Report:; 2018.

18. Fish R, MacLennan S, Alkhaffaf B, Williamson PR. "Vicarious thinking" was a key driver of score change in Delphi surveys for COS development and is facilitated by feedback of results. J Clin Epidemiol. 2020;128:118-129. doi:10.1016/j.jclinepi.2020.09.028

\subsection{Declarations}

\section{Ethical Approval and Consent to Participate}

The study was given ethical approval by the North West - Greater Manchester East Research Ethics Committee (18/NW/0347) and governance approvals by Manchester University Hospitals NHS Foundation Trust.

\section{Consent for Publication}

Not applicable

\section{Availability of Data and Material}

The datasets analysed during the current study available from the corresponding author on reasonable request.

\section{Competing Interests}

The authors report no conflicts of interest.

\section{Funding}

This study is funded by the National Institute for Health Research (NIHR) Doctoral Research Fellowship Grant (DRF-2015-08-023). JMB is partially funded by the NIHR Bristol Biomedical Research Centre and the MRC ConDUCT-II Hub for Trials Methodology Research. PRW was funded by the MRC North West Hub for Trials Methodology Research (Grant ref: MR/K025635/01).

This paper presents independent research funded by the National Institute for Health Research (NIHR). The views expressed are those of the author(s) and not necessarily those of the NHS, the NIHR or the Department of Health.

\section{Acknowledgements}

The authors would like to highlight the role undertaken by Dr Aleksandra Metryka, Senior Clinical Trials Coordinator, who facilitated the running of this study. In addition, the authors would like to thank the following associations and groups for their support in facilitating recruitment to the GASTROS study Delphi survey: 
- The International Gastric Cancer Association (www.igca.info)

- The Association of Upper Gastro-Intestinal Surgeons of Great Britain and Ireland (www.augis.org)

- The Brazilian Gastric Cancer Association (www.abcg.org.br)

- The Canadian Gastric Cancer Association (www.gastriccancer.ca)

- The Chinese Gastric Cancer Association

- The Dutch Upper GI Cancer Group (www.ducg.nl)

- The GASTRODATA group (www.gastrodata.org)

- Italian Research Group for Gastric Cancer (www.gircg.it)

- The Korean Gastric Cancer Association (www.kgca-i.or.kr)

- Oesophago-Gastric Surgery Section of the Asociación Española de Cirujanos - Spain (www.aecirujanos.es)

- Upper GI International Robotic Association (www.ugira.org)

- United Kingdom Oncology Nursing Society (www.ukons.org.uk)

- The European Oncology Nursing Society (www.cancernurse.eu)

- The Oesophageal Patients Association - United Kingdom (www.opa.org.uk)

- My Gut Feeling - Canada (www.mygutfeeling.ca)

- No Stomach for Cancer - USA (www.nostomachforcancer.org)

- Vivere Senza Stomaco - Italy

- Gastro/Oesophageal Support and Help Cancer Group (Bristol) - United Kingdom

\section{GASTROS International Working Group Collaborators (To be cited as collaborators in PUBMED)}

- Ademola Adeyeye

- Paulo Matos Costa

- Ismael Diez del Val

- Suzanne Gisbertz

- Ali Guner

- Simon Law

- Hyuk-Joon Lee

- Ziyu Li

- Koji Nakada

- Daniel Reim

- Gian Luca Baiochhi

- William Allum

- Asif Chaudhry

- Ewen Griffiths

- Shuangxi Li

- Yu-long He

- Zekuan Xu

- Yingwei Xue

- Han Liang

- Guoxin Li

- Enhao Zhao

- Philipp Neumann

- Linda O'Neill

- Emer Guinan

- Daniela Zanotti

- Giovanni de Manzoni

- Eliza R.C. Hagens

- Mark I. van Berge Henegouwen

- Patrícia Lages

- Susana Onofre

- Rafael Mauricio Restrepo Nuñez 
- Gabriel Salcedo Cabañas

- Maria Posada Gonzalez

- Cristina Marin Campos

- Bahar Candas

- Bahadir Emre Baki

- Muhammed Selim Bodur

- Reyyan Yildirim

- Arif Burak Cekic

\section{Figures}

Figure 1 Countries from which participants were recruited. Eastern countries were defined as those within East Asia, South East Asia, and Eastern Russia, and included China, Japan, South Korea, Thailand, Vietnam, Malaysia, and Singapore ${ }^{9}$. Western countries were defined as those from Western Europe, North America, Australia, and New Zealand ${ }^{10}$

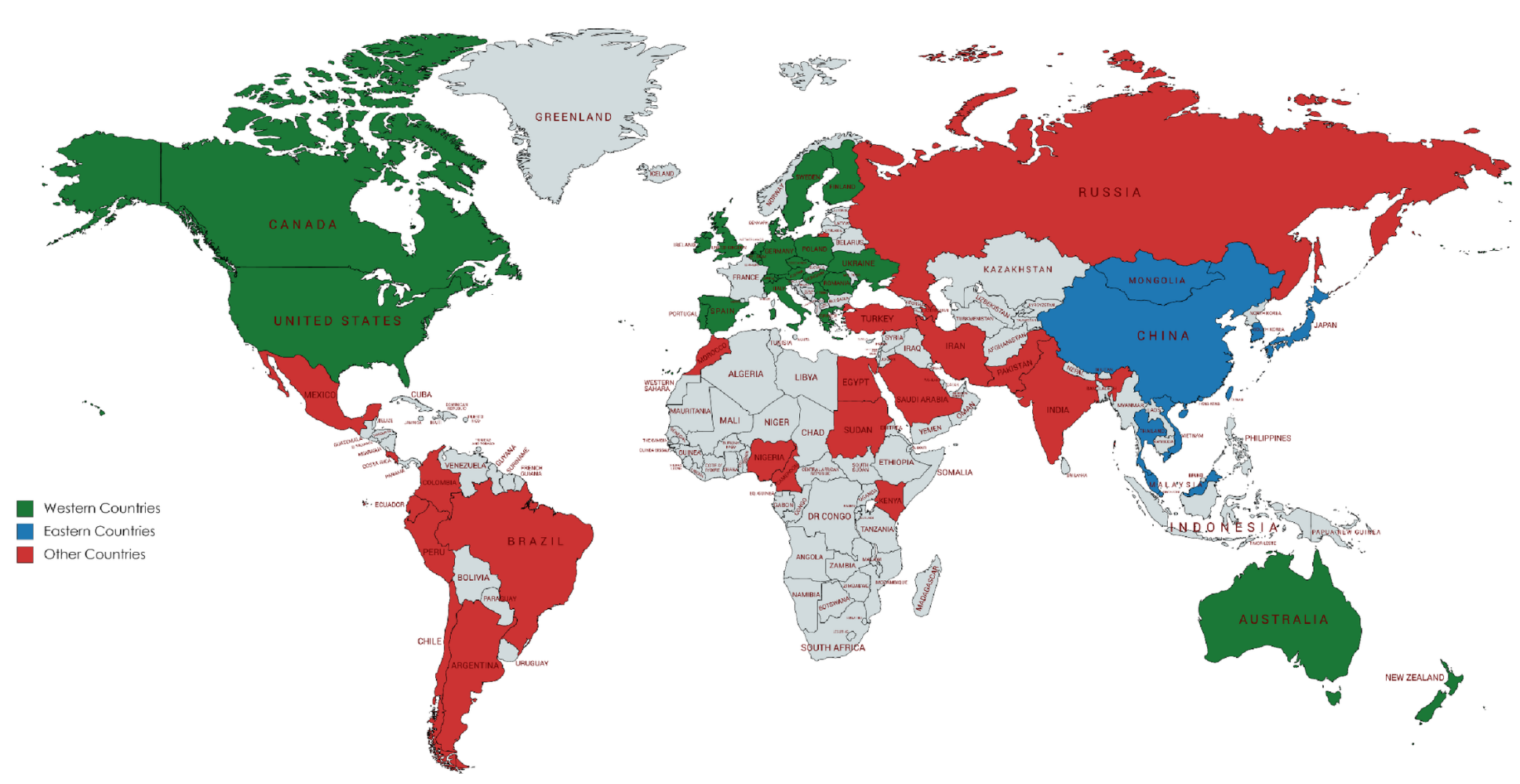

\section{Figure 1}

Countries from which participants were recruited. Eastern countries were defined as those within East Asia, South East Asia, and Eastern Russia, and included China, Japan, South Korea, Thailand, Vietnam, Malaysia, and Singapore. Western countries were defined as those from Western Europe, North America, Australia, and New Zealand. Note: The designations employed and the presentation of the material on this map do not imply the expression of any opinion whatsoever on the part of Research Square concerning the legal status of any country, territory, city or area or of its authorities, or concerning the delimitation of its frontiers or boundaries. This map has been provided by the authors. 